\title{
On the Role of Charged Aerosols in Polar Mesosphere Summer Echoes
}

\author{
John Y. N. Cho, Timothy M. Hall, Michael C. Kelley \\ School of Electrical Engineering, Cornell University, Ithaca, New York
}

\begin{abstract}
Submicron aerosols, as evidenced by the occurrence of polar mesospheric and noctilucent clouds, exist at heights from which polar mesosphere summer echoes (PMSE) are observed. We investigate the role of positively and negatively charged aerosols in the scattering processes proposed in the literature. These aerosols, if charged substantially, can account for the remarkably high radar reflectivity at both VHF and UHF by raising the electron Schmidt number through the ambipolar effect. A positively charged component may be responsible for enhanced UHF radar scatter by increasing the incoherent scatter power through a dressed dust effect, although such a process is not realistic as an explanation for VHF scatter during PMSE. Such an enhanced UHF scatter will be associated with extremely narrow backscatter spectra. We propose a model in which both negatively and positively charged aerosols are present to explain both the radar properties and the rocket probe observations of charged particle depletions. Finally, we point out that the Poker Flat 50-MHz long-term data, which contrary to accepted dynamical theory show average downward velocities in the summertime upper mesosphere, can be attributed to the fall speed of the aerosols responsible for PMSE.
\end{abstract}

\section{INTRODUCTION}

Radars operating near $50 \mathrm{MHz}$ [Ecklund and Balsley, 1981; Czechowsky et al., 1989; Röttger et al., 1990], $224 \mathrm{MHz}$ [Röttger et al., 1988], and $933 \mathrm{MHz}$ [Röttger et al., 1990] have detected extremely high backscatter cross sections from the summer polar mesosphere, a phenomenon known as polar mesosphere summer echoes (PMSE). Turbulent scatter as well as Fresnel type reflection have been invoked as the probable mechanisms, but both have encountered difficulties in explaining the smallness of the required scattering structure in the electron density. For example, in a turbulencebased scattering theory the Bragg scattering lengths seem to be well beyond the viscous cutoff point. Likewise, it seems improbable that coherent ledges with vertical length scales of the order of the radar wavelengths necessary for partial reflection could exist. Noting the occurrence of heavy, hydrated ions in the uniquely cold summer polar mesopause, Kelley et al. [1987] proposed that the diffusivity of the electrons, which are necessarily coupled to the ions through ambipolar effects, might be lowered by the existence of these relatively massive particles, thereby raising the Schmidt number which is defined by

$$
S c=\frac{\nu}{D_{e}}
$$

where $\nu$ is the kinematic viscosity of the neutral gas and $D_{e}$ is the diffusivity of electrons. The usual assumption [e.g., Hill and Bowhill, 1976] had been to take $S c \sim 1$, which implied that the diffusive cutoff length scale for the turbulent electron density fluctuation was about equal to the viscous cutoff of the neutral gas. $S c>1$ would create a viscous-convective subrange of the electron density fluctuation allowing structuring at scales shorter than the neutral cutoff. Using a test proposed by Kelley et al. [1987], Hall

\section{Copyright 1992 by the American Geophysical Union.}

Paper number 91JD02836.

$0148-0227 / 92 / 91 \mathrm{JD}-02836 \$ 05.00$ and Brekke [1988] have inferred increased electron Schmidt numbers near the summer polar mesopause from the spectral widths of incoherent scatter radar measurements. An analogous formal argument has not been made for the case of Fresnel reflection, but heuristically one can say that a decrease in the electron diffusivity would increase the lifetime of sharp, coherent ledges but would not account for ledges being created in the first place.

The effect of hydrated protons on the Schmidt number has been investigated by Hall [1990] with the conclusion that little change is effected if the polarization interaction model between ions and neutrals is adopted, whereas an ad hoc assumption of a hard sphere interaction model produces a more significant change. (The question of which model is appropriate will be discussed in the next section.) Kelley et al. [1990] have estimated $S c$ to be as high as 100 from a comparison of 53.5-MHz radar and rocket data, an enhancement which is beyond the reach of the proton hydrates.

As a possible alternative mechanism we examine here the role of charged aerosols in influencing the electron Schmidt number. This is an attractive idea since such particles are known to occur in the summer polar mesopause, as evidenced by noctilucent and polar mesospheric clouds, and their height range of occurrence is very similar [Thomas and Olivero, 1986]. There is a wide size range of aerosols, from meteor dust to the visible cloud ice crystals $(\sim 0.1 \mu \mathrm{m})$, which encompasses the various stages of cloud nucleation and coagulation processes. Even though their number density is likely very small (especially for the larger particles), we show below that they can significantly influence the motion of electrons if they are highly charged.

In addition, we shall explore the possible role aerosols play in creating an apparent downward net flow in the summer polar mesosphere as detected by radars. A downward summer mean velocity [Balsley and Riddle, 1984; Hall et al., this issue] was quite surprising but had been explained with the so-called Stokes drift effect [Coy et al., 1986]. However, in a companion paper by Hall et al. [this issue] a new calculation of the Stokes drift puts this explanation in question, and we argue here that the effect is due to falling charged aerosols. 


\section{Diffusion In THE Mesosphere}

\section{Cluster Ion and Aerosol Diffusivity}

Since the electron diffusion is tightly controlled by the heavier charged species, we first develop models suitable for such species. There are two important factors one must consider when calculating the diffusivity of charged aerosols. (1) The electric charge will induce polarization in the neutral molecules so that the interaction potential between the aerosol and the neutral molecule may depart significantly from that of a simple hard sphere approximation. (2) In the limit of very large aerosols their diffusion will be best modeled by Brownian motion. Thus we see that both the size and the charge of the aerosol directly affect its diffusivity.

In the small-scale limit, such that a charged aerosol can be viewed as an ion, the diffusivity is given by [Chapman and Cowling, 1970]

$$
D_{a}=\frac{3 k T}{16 \mu_{a n} N_{n} \Omega_{a n}}
$$

where $k$ is the Boltzmann constant, $T(\mathrm{~K})$ is the temperature (note that we are assuming thermal equilibrium between aerosols and neutrals such that $T_{a}=T_{n}=T$, which is justified at the altitudes of interest here according to Grams and Fiocco [1977]), $N_{n}\left(\mathrm{~m}^{-3}\right)$ is the neutral number density, $\mu_{a n}$ is the reduced mass given by

$$
\mu_{a n}=\frac{m_{a} m_{n}}{m_{a}+m_{n}}
$$

and $\Omega_{a n}$ is a collision integral defined by Chapman and Cowling [1970]. For the hard sphere interaction model the collision integral is given by

$$
\Omega_{a n}^{H}=\sqrt{\frac{8 k T}{\pi \mu_{a n}}} \frac{\pi}{4}\left(r_{a}+r_{n}\right)^{2},
$$

where $r_{a}$ and $r_{n}(\mathrm{~m})$ are the aerosol and effective neutral radii. (For all our calculations we shall assume spherical aerosols.) For the case where polarization interaction is dominant [Schunk, 1975],

$$
\Omega_{a n}^{P}=2.07 \times 10^{-7} \sqrt{\frac{\pi \alpha Z_{a}^{2} e^{2}}{\epsilon_{0} \mu_{a n}}},
$$

where $\alpha\left(\mathrm{m}^{-3}\right)$ is the neutral atom polarizability, $Z_{a}$ is the charge number of the aerosol, $\epsilon_{0}$ is the vacuum permittivity, and $e$ is the elementary charge. The corresponding diffusivities are then given by

$$
\begin{aligned}
D_{a}^{H} & =\frac{3}{8} \sqrt{\frac{k T}{2 \pi \mu_{a n}}} \frac{1}{N_{n}\left(r_{a}+r_{n}\right)^{2}}, \\
D_{a}^{P} & =\frac{9.06 \times 10^{5} k T}{N_{n}\left|Z_{a}\right| e} \sqrt{\frac{\epsilon_{0}}{\pi \alpha \mu_{a n}}} .
\end{aligned}
$$

For ion-neutral collisions the polarization model has been shown to be fairly good [Dalgarno et al., 1958], so we expect this to hold for the limit of small aerosols. However, in the limit of very large size (holding $Z_{a}$ constant), one might expect, since the polarization potential falls off as $r^{-4}$, that the collisions will occur as hard spheres before the neutral molecules have approached close enough to become polarized. Thus we may estimate the transition size where the hard sphere model takes over from the polarization model by equating the values of their collision integrals:

$$
\Omega_{\text {an }}^{H}=\Omega_{\text {an }}^{P} .
$$

Solving for the critical radius, we get

$$
r_{a c}=4.55 \times 10^{-4}\left(\frac{2 \alpha Z_{a}^{2} e^{2}}{\epsilon_{0} k T}\right)^{\frac{1}{4}}-r_{n} .
$$

For the summer polar mesopause we take $\alpha=1.76 \times$ $10^{-18} \mathrm{~m}^{-3}$ (corresponding to $\mathrm{N}_{2}$ which is the main neutral constituent), $r_{n}=1.8 \times 10^{-10} \mathrm{~m}$, and $T=130 \mathrm{~K}$, which gives, for a single charge,

$$
r_{a c}=5.2 \times 10^{-10} \mathrm{~m} \text {. }
$$

For hydrated protons, which can cluster into fairly large sizes, we can crudely estimate (following Hall [1990]) that the volume is proportional to the hydration number. If one assumes a hexagonal ice lattice structure where a unit cell containing 4 molecules has a volume of $1.3 \times 10^{-28} \mathrm{~m}^{3}$ [Michel, 1978], then the effective volume occupied per water molecule is about $3.3 \times 10^{-29} \mathrm{~m}^{3}$. Consequently, about 20 water molecules will fill up a spherical volume with the critical radius. This is an extremely rough estimate, but it helps to shed some light on the question of applicability of the hard sphere model to highly hydrated protons, as discussed by Kelley et al. [1987] and Hall [1990]; that is, the polarization model should be used for ions with hydration number less than 20. Kelley et al. [1987] have already pointed out that the polarization model gives a mass dependence which is too weak to have a significant impact on the electron Schmidt number. Since there are probably very few ions with hydration number over 20 , we can also eliminate the importance of the size effect of the hard sphere model. Thus we conclude that the hydrated protons cannot have a significant impact on raising the electron Schmidt number.

Going back to the consideration of aerosols, we look to the limit of large size where they can no longer be studied in the context of kinetic theory but rather must be thought of as a macroscopic particle in a continuum. This transition is characterized by the Knudsen number defined by

$$
K n=\frac{\lambda_{n}}{r_{a}}
$$

where $\lambda_{n}$ is the mean free path of the neutral molecules given by

$$
\lambda_{n}=\frac{1}{4 \sqrt{2} \pi r_{n}^{2} N_{n}} .
$$

Taking $N_{n}=1.94 \times 10^{20} \mathrm{~m}^{-3}$ for mesopause altitudes [Hall, 1990], we get $\lambda_{n}=9 \mathrm{~mm}$. This is much larger than the radius of the biggest aerosols in the mesopause region, so $K n \gg 1$, and we conclude that the aerosols are in the free molecular flow regime, unlike those of similar size in the troposphere that are in the continuum flow regime. Thus the hard sphere diffusivity should be a good model even for the largest particles under our consideration.

\section{Electron Diffusivity}

Diffusive motion of the electrons is normally taken to be directly coupled to that of the ions through the ambipolar electric field effect. However, direct application of the ambipolar diffusion equations to aerosols is not justified since their number density is much smaller than that of the ions and electrons. Thus at first glance one may tend to dismiss 
the effect of the aerosols on electron diffusion as negligible. We shall proceed to show that this is not necessarily true.

Hill [1978] developed a theory for ambipolar diffusion in a multiconstituent, weakly ionized plasma that is applicable to the $D$ region. He derived a set of coupled partial differential equations for the case of quasi-neutral (i.e., for length scales much larger than the electron Debye length) diffusion of three singly charged species: electrons, positive ions, and negative ions. Magnetic field effects are ignored, and the perturbation terms are assumed small enough for linearization. We generalize the equations such that we can accommodate electrons, positive ions, and multiply charged aerosols of either sign. (We are ignoring negative ions since they seem to be scarce in the summer mesopause region [Björn et al., 1985].)

$$
\begin{gathered}
\frac{\partial n_{c}}{\partial t}=\frac{\left|Z_{a}\right| D_{+}+Z_{+} D_{a}}{Z_{+}+\left|Z_{a}\right|} \nabla^{2} n_{c}+ \\
{\left[\frac{D_{+}-D_{a}}{Z_{+}+\left|Z_{a}\right|}+\left(1-\frac{N_{a}}{N_{e}} Z_{a}\right) D_{+}-\frac{N_{a}}{N_{e}}\left|Z_{a}\right| D_{a}\right] \nabla^{2} n_{e}} \\
\frac{\partial n_{e}}{\partial t}=\frac{\left|Z_{a}\right| D_{+}-Z_{+} D_{a}}{Z_{+}+\left|Z_{a}\right|} \nabla^{2} n_{c}+ \\
{\left[\frac{D_{+}+D_{a}}{Z_{+}+\left|Z_{a}\right|}+\left(1-\frac{N_{a}}{N_{e}} Z_{a}\right) D_{+}+\frac{N_{a}}{N_{e}}\left|Z_{a}\right| D_{a}\right] \nabla^{2} n_{e}}
\end{gathered}
$$

where $n_{c}=n_{+} \pm n_{a}$ is the sum/difference of the perturbation densities of the positive ions and aerosols (plus for negatively charged aerosols and minus for positively charged aerosols), $n_{e}$ is the perturbation density of the electrons, $Z_{+}$and $Z_{a}$ are the charge numbers on the positive ions and aerosols, $D_{+}$ and $D_{a}$ are the diffusion coefficients of the positive ions and aerosols, and $N_{a} / N_{e}$ is the ratio of the background densities of the aerosols to the electrons. It is a simplified model since it admits only one size and charge state of aerosols, whereas really there is a continuous range in their size distribution. Keeping this in mind we proceed with a numerical analysis of the equations.

If we define an effective electron diffusion coefficient $D_{e}^{\text {eff }} \equiv 1 / \tau \kappa^{2}$ for a sinusoidal perturbation with wavenumber $\kappa$ and an exponential decay time constant $\tau$, we can run a computer simulation of the equations with appropriate initial conditions and measure $\tau$ to get $D_{e}^{\text {eff }}$. There is no compelling reason to expect $D_{e}^{\text {eff }}$ to be dependent on $\kappa$. The problem has the free parameters $Z_{+}, Z_{a}, D_{+}, D_{a}$, and $N_{a} / N_{e}$. We will take $Z_{+}=1$ for convenience and use the ratio $D_{a} / D_{+}$as a single parameter. Using the arguments made in the previous section and assuming (1) $m_{a} \gg m_{n}$, (2) the ions are dominated by polarization interaction, and (3) the aerosols are well characterized by the hard sphere model ( $r_{a} \gg r_{+}$, the effective radius of the positive ions), we can derive an expression for this parameter

$$
\frac{D_{a}}{D_{+}}=4.14 \times 10^{-7} \sqrt{\frac{\alpha \mu_{+n}}{2 \epsilon_{0} k T m_{n}}} \frac{e}{r_{a}^{2}},
$$

where $\mu_{+n}$ is the reduced mass of the positive ion and neutral molecule. Plugging in our mesopausal parameters and using the mass of $\mathrm{H}^{+}\left(\mathrm{H}_{2} \mathrm{O}\right)_{5}$, which from Björn et al. [1985] is a representative ion species at $85 \mathrm{~km}$, we get

$$
\frac{D_{a}}{D_{+}}=4.3 \times 10^{-19} \tau_{a}^{-2},
$$

where $r_{a}$ is in meters. Once we fix $r_{a}$, the only free parameters are $Z_{a}$ and $N_{a} / N_{e}$.

Figures $1 a$ to $1 d$ show the results of our simulations for $D_{e}^{\text {eff }} / D_{+}$plotted versus $N_{a} / N_{e}$. Multiple curves represent the different values of $D_{a} / D_{+}$that are determined by the indicated values of $r_{a}$. The consecutive figures correspond respectively to the aerosol charge $Z_{a}=-100,-10,10,100$. Since the usual assumption has been to take $D_{e} / D_{+}=2$ (the ambipolar result) where $D_{+}$is given by the dominant ion constituent, we will give our results in terms of the ratio $D_{e}^{\text {eff }} / D_{+}$so that it is easily compared to the base result of 2 . As one would expect, the curves go from $D_{e}^{\text {eff }} / D_{+}=2$ (the standard ambipolar diffusivity), with no aerosols present, to $D_{e}^{\text {eff }} / D_{+}$approaching $D_{a} / D_{+}$as the aerosols become numerous. Notice that the curves in Figure $1 a$ are virtually indistinguishable from those in Figure $1 b$. This is also true for Figures $1 c$ and $1 d$. Since the $x$ axes differ by a factor of 10 , it seems that the important scaling factor is not $N_{a} / N_{e}$ but $\left|Z_{a}\right| N_{a} / N_{e}$. This makes sense physically; it means that the important factor is how much charge of the plasma is tied up in aerosols. Also, for the larger aerosols of our interest the transition from ion to aerosol domination occurs sharply around $\left|Z_{a}\right| N_{a} / N_{e}=1.2$ (for $Z_{a}$ negative) and 0.6 (for $Z_{a}$ positive), in other words, when somewhat more than half of the charge is tied up in aerosols. Calculating $D_{+}$for the cluster ions from (7) and using (1), we get $S c=1$ for $D_{e}^{\text {eff }} / D_{+}=2.8$, so it follows that $S c=100$ for $D_{e}^{\text {eff }} / D_{+}=0.028$ and $S c=1000$ for $D_{e}^{\text {eff }} / D_{+}=0.0028$. These values of $S c$ are shown as well. One can conclude from Figures $1 a$ through $1 d$ that in order for the electron Schmidt number to reach $100, r_{a}$ must be at least $0.01 \mu \mathrm{m}$ and $\left|Z_{a}\right| N_{a} / N_{e}$ must be greater than 1.2 (for $Z_{a}$ negative) and greater than 0.6 (for $Z_{a}$ positive). The same conditions hold for the Schmidt number to reach 1000 except that $r_{a}$ must be at least $0.03 \mu \mathrm{m}$ since $S c$ scales as $r_{a}{ }^{-2}$.

\section{On the Charge State of Aerosols IN THE MESOSPHERE}

We now have a model for raising the electron Schmidt number through the ambipolar influence of charged aerosols. Realistic values for the parameters must now be estimated to evaluate their effect on several mesospheric processes using this model. The size range of the aerosols is fairly well known. The largest cloud particles are associated with noctilucent clouds and are believed to be $\sim 0.3 \mu \mathrm{m}$ [Turco et al., 1982]. Polar mesospheric clouds are thought to be due to smaller aerosols of the order of 0.05-0.1 $\mu \mathrm{m}$ [Thomas, 1984].

Unfortunately, there is a great deal of uncertainty associated with the aerosol number density $N_{a}$. Observed values range from $10^{6} \mathrm{~m}^{-3}$ [Bronshtehn and Grishin, 1976] to $1.5 \times 10^{8} \mathrm{~m}^{-3}$ [Thomas, 1984] for $r_{a}=0.07 \mu \mathrm{m}$, while a model calculation by Turco et al. $[1982]$ gives $<10^{7} \mathrm{~m}^{-3}$ for $r_{a}>0.05 \mu \mathrm{m}$. For the sake of simplicity we will assume a value of $N_{a}=10^{7} \mathrm{~m}^{-3}$ for all further calculations. Using $r_{a}=0.06 \mu \mathrm{m}$ and $N_{e}=3 \times 10^{9} \mathrm{~m}^{-3}$ as typical values for the summer mesopause, in order to reach the critical ratio required for PMSE of $\left|Z_{a}\right| N_{a} / N_{e} \approx 1.2$ (for negative $Z_{a}$ ), $Z_{a}$ must be $\approx-360$. To reach $\left|Z_{a}\right| N_{a} / N_{e} \approx 0.6$ (for positive $\left.Z_{a}\right), Z_{a}$ must be $\approx 180$. Several rocket flights during PMSE events have indicated electron density bite-outs at the radar scattering heights [Ulwick et al., 1988; Inhester et al., 1990]. An example from the latter paper is reproduced in Figure 2 

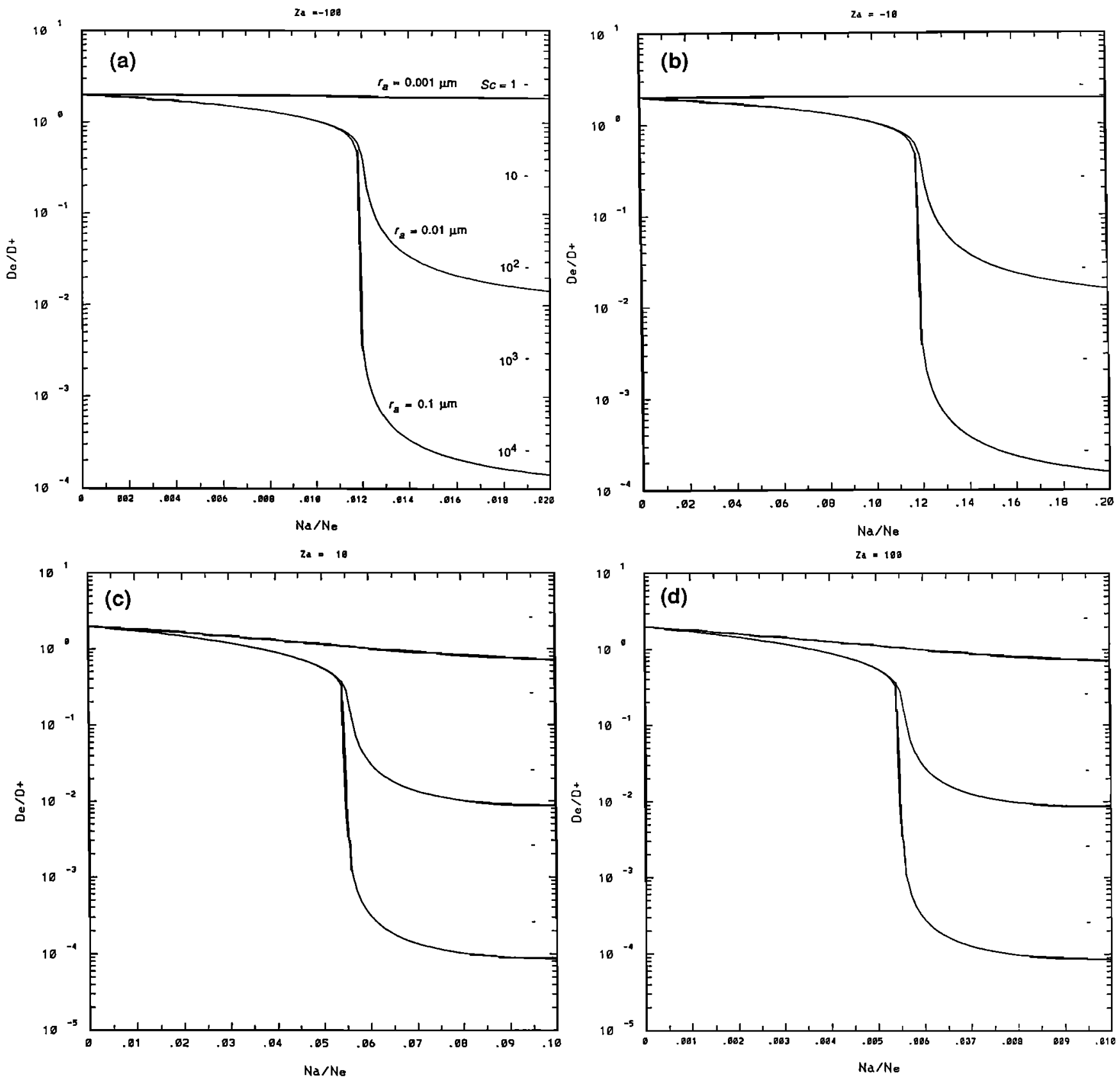

Fig. 1. Plot of effective electron diffusivity versus aerosol number density with (a) $Z_{a}=-100,(b) Z_{a}=-10$, (c) $Z_{a}=10$, and $(d) Z_{a}=100$. Electron diffusivity is normalized with respect to the positive ion diffusivity, and the aerosol number density is normalized with respect to electron number density.

(curve labeled $r f$ probe) and shows reduction of $N_{e}$ to a value $\approx 3.5 \times 10^{8} \mathrm{~m}^{-3}$. In this case, the corresponding critical values of $Z_{a}$ are -42 and 21 .

Are these plausible values? There have been no measurements of mesospheric aerosol charge, but calculations involving charge fluxes to aerosol particles by Turco et al. [1982] yield an estimate of $Z_{a} \sim-4$. So only the combination of a large electron density depletion and the highest estimate of large aerosol density would seem to lead to a significant change in the electron diffusivity. This seems to be a problem since some intense PMSE events occur without bite-outs (e.g., the Structure and Atmospheric Turbulence Environment (STATE) 1 case presented by Ulwick et al. [1988]).
However, it has been pointed out by Havnes et al. [1990] that the photoionization effect, usually neglected due to the high work function for pure ice, could be dominant over collisional charging since the aerosol surface is likely contaminated by metallic substances from meteor vapor which have much lower work functions. Photoemission would lead to positively charged aerosols which have the possibility of a much higher state than the case for negative charging. This can be understood as follows. The voltage associated with a charged object equals the charge divided by the capacity. For the same size aerosol, $C$ is fixed and $Q \propto V$. An electrically "floating" object subject to a flux of electrons and positive species will charge negatively to a potential propor- 


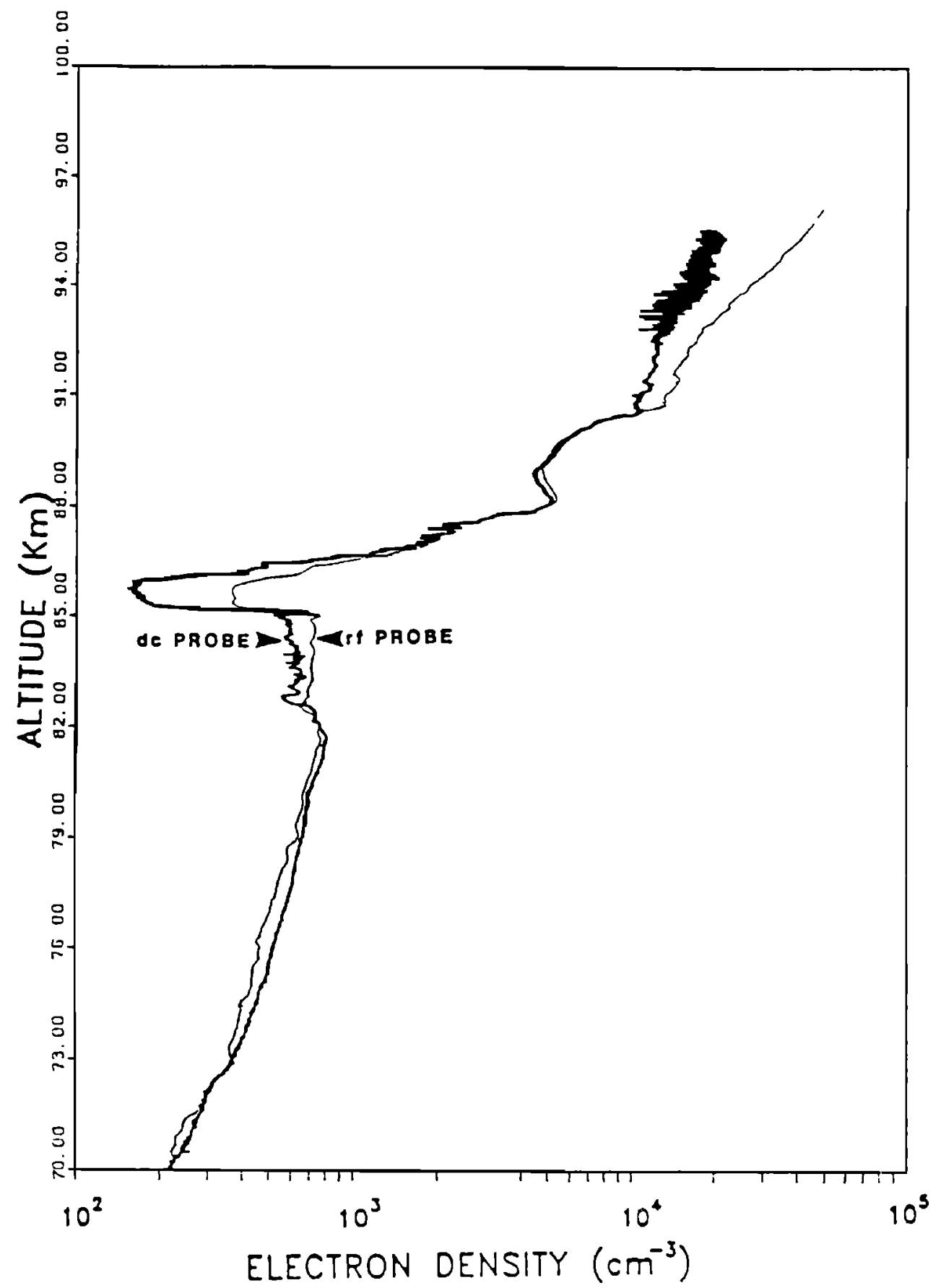

Fig. 2. Rocket-borne electron density measurement versus height [from Inhester et al., 1990]. The dark trace is the DC Langmuir probe response and the light trace is the RF capacitive probe data. This rocket was launched on July 14, 1989, from the Andøya Rocket Range in Norway while PMSE were being received by the sounding system (SOUSY) 53.5-MHz radar.

tional to $k T_{e} / e$, provided that $T_{e}$ is well below a value at which secondary emission can occur. In the polar summer mesosphere, $k T_{e} / e \approx 0.01 \mathrm{~V}$. If photoemission is allowed, however, and if the electron density is low, then the object will charge positively to a value proportional to the "temperature" of the solar UV flux which is typically taken to be $5 \mathrm{eV}$ [Goertz, 1989]. The positive charge state thus could be 100 times the negative state.

The ultimate upper bound for the amount of charge that an aerosol can sustain is dictated by the electrostatic fragmentation criterion, i.e., the point at which the electrostatic stress exceeds the tensile strength of the material. For a sphere with a homogeneously charged surface, the critical electric field strength is [Böhnhardt, 1986]

$$
E_{c}=\sqrt{\frac{2 \sigma}{\epsilon}},
$$

where $\epsilon$ is the permittivity and $\sigma$ is the tensile strength of the aerosol material. Theory predicts $\epsilon$ proportional to $T^{-1}$ and experiment gives $\epsilon \approx 2.2 \times 10^{-7} T^{-1}$ for pure ice [A uty and Cole, 1952]. Thus for $T=130 \mathrm{~K}, \epsilon \approx 1.5 \times 10^{-9} \mathrm{~F} / \mathrm{m}$. The corresponding critical charge number is 


$$
\left|Z_{c}\right|=\frac{4 \pi r_{a}^{2}}{e} \sqrt{2 \epsilon \sigma} .
$$

Michel [1978] gives the tensile strength of polycrystalline ice to be

$$
\sigma=7.94 \times 10^{3} \sqrt{\frac{1-9 \times 10^{-4}(T-273)}{2 r_{a}}} \mathrm{~N} / \mathrm{m}^{2}
$$

which yields

$$
\left|Z_{c}\right|=3.3 \times 10^{17} r_{a}^{\frac{3}{2}}
$$

for our parameters. For $r_{a}=0.06 \mu \mathrm{m},\left|Z_{c}\right|=5 \times 10^{6}$. This is probably an overestimate because the tensile strength is likely to be lower than the model value. But the point is that the real limit to the amount of charge carried by an aerosol is not the fragmentation criterion but the balance of charge flux to the aerosol, photoemission, and charge exchange collisions.

Our diffusion model does not distinguish sharply between positive and negative $Z_{a}$. In light of the past model charging calculations, highly negative charge is ruled out; thus positive charging by a dominant photoionization effect becomes the attractive alternative. On the other hand, as mentioned earlier, we have evidence from rocket flights [ $\mathrm{Ul}$ wick et al., 1988; Kelley et al., 1990] that sometimes there is a significant electron density bite-out around the PMSE height range which is most easily explained by the scavenging of electrons by a layer of aerosols (which would then be negatively charged). Reid [1990] examines this scenario and concludes that the aerosols doing the scavenging must have radii of the order of $0.01 \mu \mathrm{m}$ or less due to the small number of larger particles and the necessarily low negative charge on the aerosols. A totally different explanation proposed by Havnes et al. [1990] is that an apparent reduction in the electron density could result from the inability of the DC Langmuir probes (used in all the rockets) to deflect massive, positively charged aerosols. The probes measure a current proportional to

$$
N_{e}-Z_{a} N_{a}=N_{+} .
$$

In other words, the bite-out is of the positive ions rather than of the electron density. However, data from a rocket carrying both a Langmuir probe and an RF capacitive probe reveal a bite-out for both probes, but with a noticeably larger magnitude for the latter in that height range (see Figure 2). Since the RF probe would not inadvertently measure positively charged aerosols, the pure ion bite-out explanation would seem to be proven wrong. Furthermore, without steep edges in the electron density it seems unlikely that the radar echo characteristics that point toward a specular reflection mechanism could be explained.

One way to interpret these data is to invoke both of the above bite-out explanations; i.e., the smaller aerosols are scavenging electrons, thus creating a real depletion in electron density, with positively charged larger aerosols ramming into the Langmuir probe to create a deeper apparent bite-out. Clearly, this proposition requires that larger aerosols be preferentially charged positively. For positively charged aerosols with $r_{a} \leq 0.3 \mu \mathrm{m}$, the equation for current equilibrium between aerosol and environment is [Havnes et al., 1990]

$$
\begin{aligned}
N_{e} \overline{c_{e}}\left(1+\frac{Z_{a} e^{2}}{4 \pi \epsilon k T r_{a}}\right)= & N_{\mathrm{c}} \overline{c_{1}} \exp \left(-\frac{Z_{a} e^{2}}{4 \pi \epsilon k T r_{a}}\right) \\
& +3 \times 10^{4} \gamma F_{\nu} r_{a} \mathrm{~m}^{-2} \mathrm{~s}^{-1}
\end{aligned}
$$

where $\gamma$ and $F_{\nu}$, both independent of aerosol size, are the photoelectric yield and photon flux. Ignoring the small ion charge exchange term (first term on the right-hand side) and comparing the rate of electron capture (left-hand side) with the rate of photoionization (last term), we see that the latter grows linearly with $r_{a}$ whereas the former decreases with $r_{a}$. Therefore there is good reason to surmise that large aerosols are more positively charged than the smaller ones. Using this theory, we can try to glean some information from the rocket data. Estimating the difference between the plots of the two probes at the bite-out to be $\left|Z_{a}\right| N_{a}=2 \times 10^{8} \mathrm{~m}^{-3}$ and $N_{e}=3.5 \times 10^{8} \mathrm{~cm}^{-3}$, we get $\left|Z_{a}\right| N_{a} / N_{e}=0.67$. This is over the threshold value of $\sim 0.6$ that we need for the electron diffusivity to be dominated by the aerosols.

\section{Applications of the Results}

\section{Radar Scattering Effects of Aerosols}

Both the turbulent and Fresnel type scatter are coherent (i.e., not thermal) mechanisms. Incoherent scatter (resulting from random thermal motion) had been previously ruled out as a cause of PMSE since the calculated cross section for the known electron density was much too low. However, Havnes et al. [1990] have proposed that the presence of highly positively charged aerosols would boost the incoherent scatter cross section enough to account for the echo powers of PMSE at all the observed radar frequencies.

We can easily show that this idea is only applicable to the highest frequency radars with which PMSE are observed [Röttger et al., 1990]. First, the incoherent scatter cross section is not wavelength dependent for scales much larger than the electron Debye length $(\sim 1 \mathrm{~cm}$ for the summer mesopause), whereas the observed radar cross sections are strongly dependent on wavelength. A simultaneous threeradar observation of PMSE needs to be conducted in order to obtain definitive data on the frequency dependence of the radar reflectivity. The best we can do at present is to plot the maximum reported backscatter volume reflectivity at $46.9 \mathrm{MHz}, 224 \mathrm{MHz}$, and $933 \mathrm{MHz}$. This is done in Figure 3; the extremely strong wavelength dependence is clear. Second, the absolute value of the VHF reflectivity is orders of magnitude above "normal" incoherent scatter levels. In addition, Figure 3 shows an example of the calculated radar volume reflectivity versus radar scattering wavenumber for three values of Sc. The curves are calculated from the turbulence model of Driscoll and Kennedy [1985] at the same electron density, electron gas scale height of $1 \mathrm{~km}$, and with the turbulence parameter $\epsilon=1 \mathrm{~W} / \mathrm{kg}$ which is a high value for the mesosphere. The 224- $\mathrm{MHz}$ radar was not operating when the high points for $46.9 \mathrm{MHz}$ and $933 \mathrm{MHz}$ were recorded while in simultaneous observation, so it may not be possible to fit one theoretical curve through all three. Keeping this caveat in mind, note that the theoretical curve for $S c=1$ falls well short of the observed echo strengths at all three frequencies. (The parameters, i.e., electron density, electron gas scale height, and turbulent energy dissipation rate, have been pushed to the bounds of plausibility, since we are trying to match to the highest observed values of 


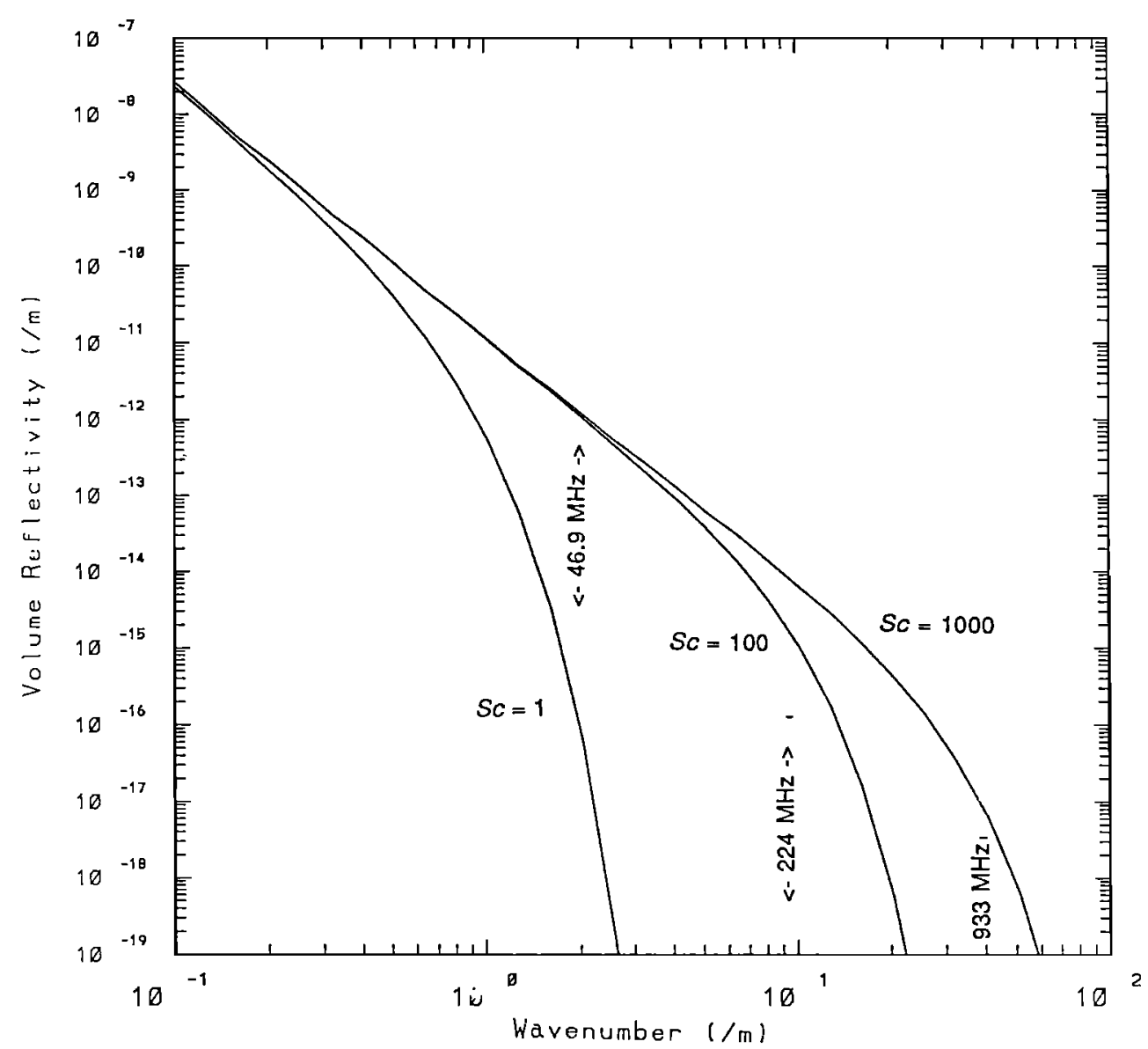

Fig. 3. Turbulent radar volume reflectivity calculated from the model of Driscoll and Kennedy [1985] with $N_{e}=8 \times 10^{9} \mathrm{~m}^{-3}$, electron scale height $=1 \mathrm{~km}$, and turbulent energy dissipation rate $=1 \mathrm{~W} / \mathrm{kg}$. The data points correspond to the highest recorded echo powers at $46.9 \mathrm{MHz}$ (CUPRI), $224 \mathrm{MHz}$ (EISCAT VHF), and $933 \mathrm{MHz}$ (EISCAT UHF).

radar reflectivity.) Also note that the curve corresponding to $S c=100$ matches fairly well to the $46.9-\mathrm{MHz}$ and $224-$ $\mathrm{MHz}$ points but falls many orders of magnitude below that of the 933-MHz mark. (Indeed, it falls well below the reflectivity of normal incoherent scatter at this point.) On the other hand, the $S c=1000$ curve fits the $46.9-\mathrm{MHz}$ and 933- $\mathrm{MHz}$ points while overshooting the $224-\mathrm{MHz}$ mark. One interpretation is to ignore the 224- $\mathrm{MHz}$ discrepancy and take $S c=1000$. Another would be to take $S c=100$ and explain the $933-\mathrm{MHz}$ PMSE as the result of enhanced incoherent scatter due to charged aerosols.

We prefer the latter argument and apply the turbulent scatter mechanism with $S c \sim 100$ to 46.9 and $224 \mathrm{MHz}$, invoking a different mechanism for the 933-MHz PMSE. Havnes et al. [1990] have applied a dusty plasma theory of Tsytovich et al. [1989] which predicts an enhancement in the incoherent radar scattering cross section by $Z_{a}{ }^{2}$. A more careful analysis by Hagfors [1991] shows that the scatter cross section due to charged aerosols is

$$
\eta_{a}=\frac{Z_{a}^{2} \sigma_{e} N_{a}}{\left(2+\beta^{2}-Z_{a} \frac{N_{a}}{N_{e}}\right)^{2}}
$$

for $\left|Z_{a}\right|>69 T^{\frac{1}{2}} N_{a}^{-\frac{1}{6}}$ and

$$
\eta_{a}=\frac{Z_{a}^{2} \sigma_{e} N_{a}}{\left(2+\beta^{2}-Z_{a} \frac{N_{a}}{N_{e}}\right)\left(2+\beta^{2}-Z_{a} \frac{N_{a}}{N_{e}}+Z_{a}{ }^{2} \frac{N_{a}}{N_{e}}\right)}
$$

for $\left|Z_{a}\right|<69 T^{\frac{1}{2}} N_{a}-\frac{1}{6}$ where $\sigma_{e}=1.0 \times 10^{-28} \mathrm{~m}^{2}$ is the scattering cross section of a single electron, $\beta=k_{R} \lambda_{D}, k_{R}$ $\left(\mathrm{m}^{-1}\right)$ is the radar scattering wavenumber, and

$$
\lambda_{D}=69\left(\frac{T}{N_{e}}\right)^{\frac{1}{2}} \mathrm{~m}
$$

is the electron Debye length. Physically, (23) applies when the aerosol separation distance is greater than the aerosol Debye length such that their self-interactions can be ignored. Equation (24) is used when the aerosols must be treated as a continuum fluid.

Comparing the two expressions to the normal D region incoherent scatter cross section of Dougherty and Farley [1963]

$$
\eta=\frac{1+\beta^{2}}{2+\beta^{2}} \sigma_{e} N_{e}
$$

we see that significant enhancement of scattered power is only possible in the first case. Thus for $N_{a}=10^{7} \mathrm{~m}^{-3}$, $\left|Z_{a}\right|$ must exceed $\sim 50$ for enhanced scatter. Röttger et al. [1990] have reported a 10-dB enhancement over the ambient incoherent scatter power (Figure 4) for the one published PMSE event at $933 \mathrm{MHz}$. According to the calibration of Röttger et al. [1990], $N_{e}=4 \times 10^{9} \mathrm{~m}^{-3}$, which yields $\beta=$ 0.5. If we take $N_{a}=10^{7} \mathrm{~m}^{-3}$, a comparison of (23) and (26) shows we need $Z_{a}=95$ or $Z_{a}=-120$ to yield a tenfold enhancement in radar reflectivity. This is a crude estimate of 


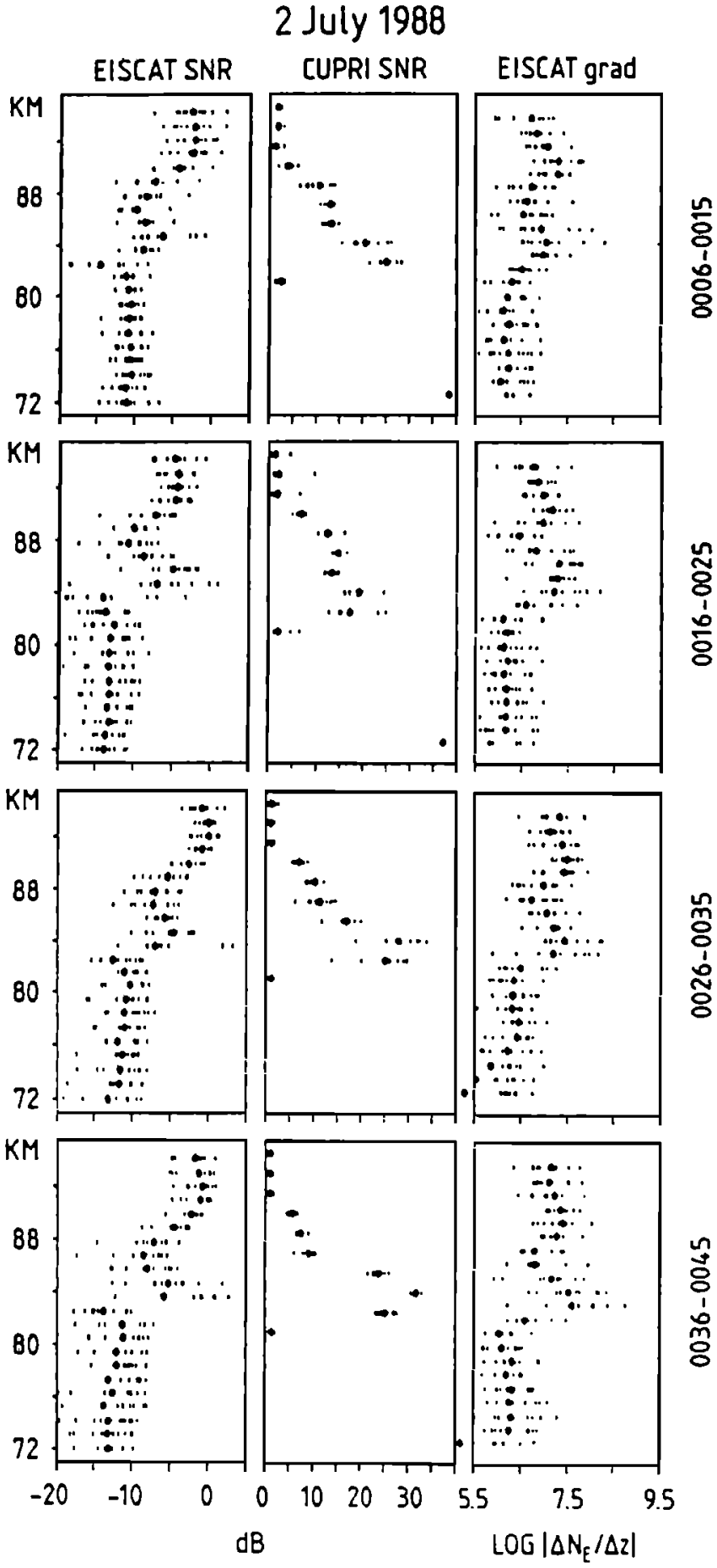

Fig. 4. Signal-to-noise ratio versus height for the time period 0006-0045 UT when a PMSE event was observed with the EIS CAT 933-MHz radar (left panels) [Rottger et al., 1990]. Center panels show CUPRI $46.9-\mathrm{MHz}$ data, and the right panels are a measure of the vertical gradient in electron density as deduced from the EISCAT data. The small dots are averaged over $1 \mathrm{~min}$, and the large dots are averaged for the indicated time per panel.

the aerosol charge required for enhanced incoherent scatter at $933 \mathrm{MHz}$. More experimental data on $Z_{\mathrm{a}}$ and $N_{\mathrm{a}}$ are needed to test the validity of this model.

As a possible source of more information we turn to the spectral width of this enhanced incoherent scatter mode. The spectral width should be inversely proportional to the time taken by the aerosol to diffuse $\lambda_{R} / 4 \pi$, where $\lambda_{R}$ is the radar wavelength. Following the approach of Fukuyama and Kofman [1980], we calculate the incoherent scatter spectral width to be

$$
\Delta f=\frac{\kappa_{R}^{2}}{\pi} \frac{2+\beta^{2}}{1+\beta^{2}} D_{a} .
$$

This expression assumes scattering from only one size of aerosols and is not valid for $\beta^{2} \gg 1$, i.e., if $\lambda_{R} / 4 \pi$ is much less than the radius of the electron cloud shielding the aerosol. (In any case, the scattered power drops off drastically (to the inverse fourth power) for large $\beta$.)

Figure 5 shows the variation of the spectral width with aerosol radius for polar summer mesopause parameters. Realistically, the electrons will enshroud aerosols and ions of various size such that the resulting spectrum would be a sort of superposition of spectra produced by the entire range, so that the spectral information would be smeared out. If, however, the electrons are divided between the positive ions and one fairly well defined size of aerosol, one should be able to see the contributions from each in the spectra: the aerosol portion should be much narrower. This is analogous to the technique used in extracting ion composition information from conventional incoherent scatter data [e.g., Farley et al., 1967]. As one moves into a region where dressed aerosol scattering becomes important, one would expect to see a narrow spectral peak starting to poke out of the normal, broad, incoherent scatter spectrum. If the aerosols are completely dominant, then the broad component should disappear altogether. If the vertical resolution of the radar is good enough, one may even observe the narrowing of the aerosol component with descending height corresponding to an increase in size due to sedimentation. However, one would not expect to see the extremely narrow spectra using a typical $\mathrm{D}$ region incoherent scatter radar mode, since such a great number of time lags need to be measured. For example, the European incoherent scatter (EISCAT) $933-\mathrm{MHz}$ data clearly show a narrowing of the spectral width in the PMSE height bin, but due to the applied radar program it was not possible to resolve widths less than $20.4 \mathrm{~Hz}$ [Röttger et al., 1990].

\section{On the Mean Downward Velocity in the Polar Summer Mesosphere}

The long-term, composite-year record from the $50-\mathrm{MHz}$ Poker Flat radar shows average downward velocities of $\sim$ $0.2 \mathrm{~m} / \mathrm{s}$ in the summertime upper mesosphere, a result that goes against the commonly accepted theoretical prediction of a much smaller velocity in the upward direction [Balsley and Riddle, 1984; Hall et al., this issue]. Coy et al. [1986] have proposed a Stokes drift explanation for this discrepancy, but the analysis by Hall et al. [this issue] in the companion paper, employing Poker Flat gravity wave spectral parameters, shows the Stokes drift to be at most $\sim 0.04 \mathrm{~m} / \mathrm{s}$. Our charged aerosol/radar scatter connection coupled with the fact that these large downward velocities are seen only during the summer and around the mesopausal height range suggests a different explanation (also see Hall et al. [this issue]). If, for example, the radio waves were scattering directly from the "aerosol-bound" electrons, the radar would measure a downward Doppler velocity corresponding to the aerosol terminal velocity. After falling to a warmer region, 


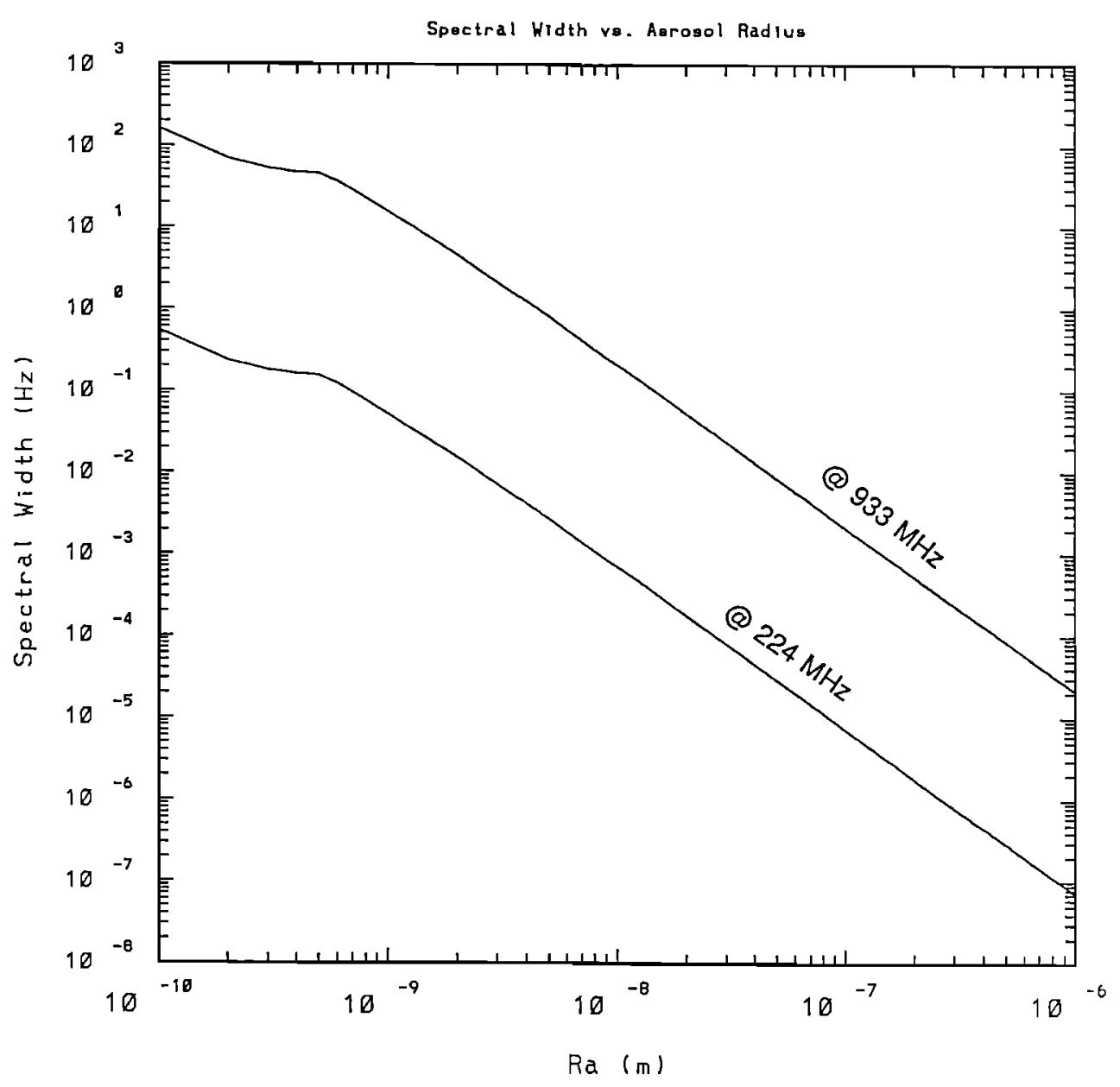

Fig. 5. Spectral width versus aerosol radius for enhanced incoherent scatter from charged aerosols. The bump near $r_{a}=5 \times 10^{-10} \mathrm{~m}$ represents the transition from the polarization to the hard sphere collision model.

they would evaporate, and the upward motion of the aerosol constituents would not be detected by the radar. In this way the background fluid motion can be in the opposite direction of the radar Doppler velocity. However, this is not exactly our situation since the radar actually scatters coherently from inhomogeneities in the electron distribution which must follow the charged aerosols. Intuitively, it seems reasonable that even though the pattern of aerosols and electrons encounter a changing dynamic input as they descend through the neutral gas, they should retain at least a partial correlation from height to height in their spatial structure, especially at scales in the viscous-convective subrange where the charged particle dynamics is effectively decoupled from the immediate behavior of the neutrals. We are planning a more rigorous analysis and a numerical simulation of this effect for a future publication.

If we take the hypothesis that the radar is indeed detecting aerosol descent, we can deduce some things about their properties. In the absence of neutral atmospheric motion the vertical velocity of a charged particle in the ionosphere where magnetic field effects are not important is given by [e.g., Kelley, 1989]

$$
\vec{v}_{a}=\frac{D_{a}}{k T}\left(Z_{a} e E-\frac{k T}{N_{a}} \frac{d N_{a}}{d z}-m_{a} g\right) \hat{z},
$$

where $E$ is the vertical electric field strength, $g$ is the grav- itational acceleration, and $z$ increases upward. First, we explore the effect of gravity alone.

Substituting (6) into (28) and assuming $m_{a} \gg m_{n}$, we solve for the aerosol radius,

$$
r_{a}=\frac{N_{n} v_{a}}{g \rho_{a}} \sqrt{\frac{8 k T m_{n}}{\pi}}
$$

where $\rho_{a}$ is the density of the aerosol $\left(920 \mathrm{~kg} / \mathrm{m}^{3}\right.$ for ice crystals). For our summer polar mesopause conditions, with $v_{a}=0.2 \mathrm{~m} / \mathrm{s}$, we get $r_{a}=0.06 \mu \mathrm{m}$, which is quite reasonable.

What about the other terms? The gradient term, $N_{a}^{-1} d N_{a} / d z$, must be at least of order unity for it to be larger than the electric term, meaning essentially, that the entire concentration of aerosols decreases to nothing over a 1-m drop. This does not seem likely, so we will ignore it. In a simple case of uniform positively charged aerosols and an equal number of electrons falling through a neutral gas, if there is no net current, then the electron fall velocity must equal the aerosol fall velocity, and ignoring the mass of electrons, we have

$$
\frac{D_{a}}{k T}\left(Z_{a} e E-m_{a} g\right)=-\frac{e D_{e}}{k T} E .
$$

Since $D_{a} / D_{e} \ll 1$, the gravitational term must indeed dominate the electrical term for plausible values of $Z_{a}$. So we are 
left with a falling charged aerosol of radius $0.06 \mu \mathrm{m}$ which is compatible with the size range of particles at the summer polar mesopause.

\section{SumMary AND Discussion}

In this paper we have developed a model in which PMSE for both VHF and UHF radars can be accounted for by the electrodynamic effect of charged aerosols. We know from optical observations of polar mesospheric and noctilucent clouds that particles with radii of $0.01-0.1 \mu \mathrm{m}$ exist in thin layers slightly below mesopause heights. Rocket measurements of electron density have revealed bite-outs at similar altitudes, and simultaneous DC Langmuir and RF capacitive probe data show a discrepancy only at those heights, which we propose could be caused by the smaller particles scavenging electrons and the larger ones, charged positively by photoemission, ramming into the Langmuir probe. Furthermore, we interpret the mean downward velocity, measured by a $50-\mathrm{MHz}$ radar during the summer in the same height region, to be the fall speed of aerosols which are responsible for the VHF PMSE. The observed velocity implies a radius of $0.06 \mu \mathrm{m}$. One can see from Figures 1a through $1 d$ that aerosols of this size, if they become dominant in the charge balance of the plasma, will impart Schmidt numbers exceed- ing 1000 to the electrons, thereby providing an explanation for the simulaneously observed highest echo power at 46.9 and $933 \mathrm{MHz}$. This could account for the spurious nature of PMSE at $933 \mathrm{MHz}$, since the turbulent scatter power at such high frequencies would only emerge above the normal incoherent scatter power level under highly enhanced geophysical conditions, i.e., high electron density and/or turbulent energy dissipation rate (see Figure 6). However, this explanation would cease to make sense if a three-frequency radar PMSE experiment shows the reflectivity at $224 \mathrm{MHz}$ to be well below that of the predicted turbulent scatter value for $S c=1000$. Such an experiment is currently planned and will be crucial in testing these ideas.

Since it is thought that negatively charged aerosols cannot exceed a charge number of about 4 , we look to a model which includes positively charged aerosols. Then, in order to achieve a significant enhancement in the electron Schmidt number to create the PMSE, we need the parameter $\left|Z_{a}\right| N_{a} / N_{e}$ to reach at least $\sim 0.6$. Estimates of $N_{a}$ vary greatly, and $N_{e}$ is also variable, depending on whether the echoes come from within the electron density bite-outs. $Z_{a} \sim 100$ would be a crude estimate. Given this high degree of aerosol charge, one is bound to see instances of enhanced incoherent scatter, as suggested by Havnes et al. [1990], with UHF radars. In this context, however, we strongly

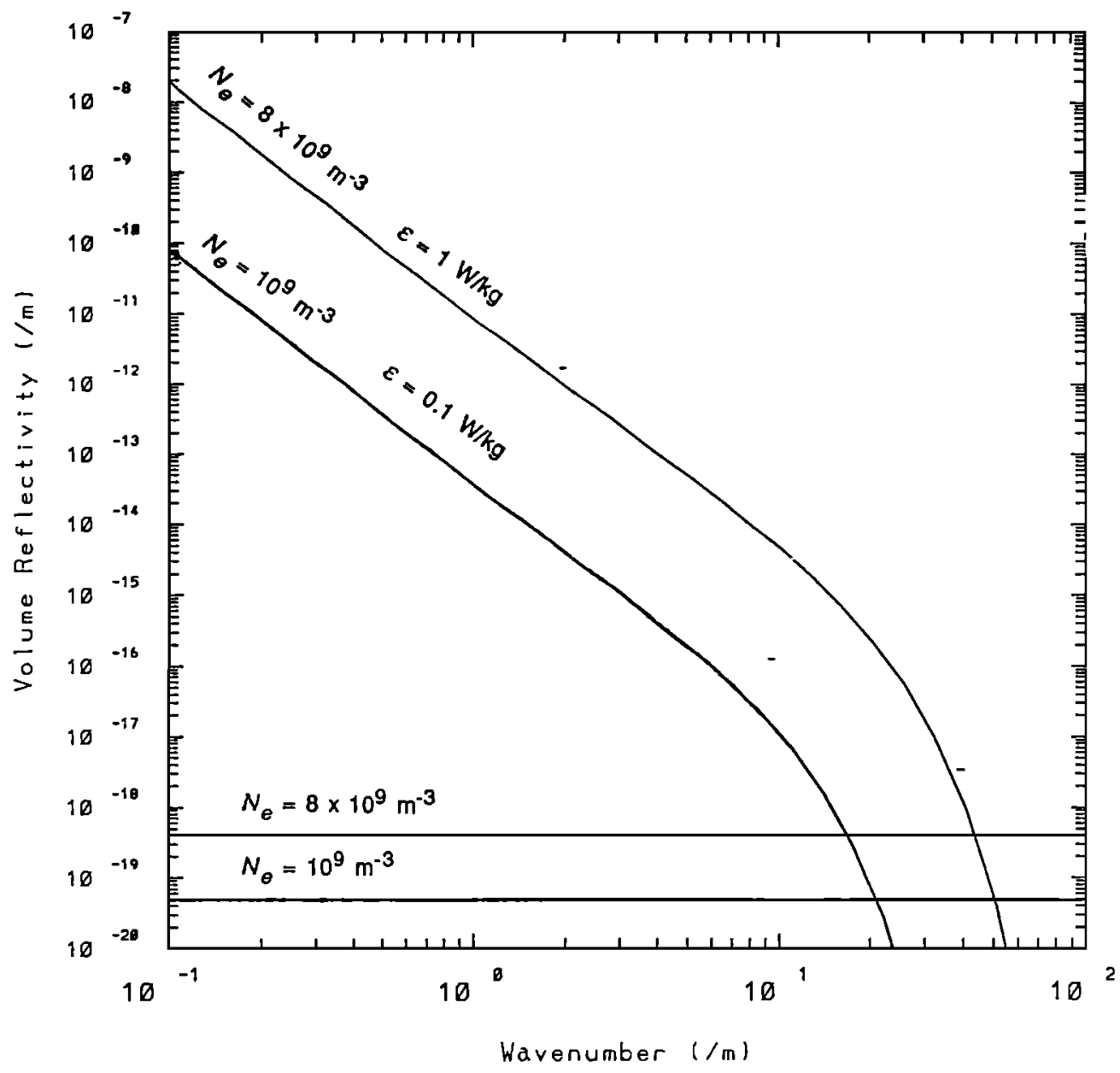

Fig. 6. The turbulent radar volume reflectivity model of Figure 1 for two values of electron density and turbulent energy dissipation rate and $S c=1000$. The two lines at the bottom are the normal incoherent scatter reflectivity (not the enhanced scatter due to charged aerosols) for the same two values of electron density. The data points are the same as in Figure 1. 
disagree with Havnes et al. [1990] that charged aerosol incoherent scatter can explain PMSE at VHF frequencies.

If it turns out that enhanced incoherent scatter is responsible for the UHF PMSE, there is no a priori reason to believe that the same aerosols are responsible for both the VHF and UHF PMSE. Power-height profiles of simultaneous PMSE at 46.9 and $933 \mathrm{MHz}$ given by $R o ̈ t t g e r$ et al. [1990] show the former occurring at a slightly lower height than the latter. (Granted, absolute altitude calibration for the Cornell University portable radar interferometer (CUPRI) was not done to within the height resolution of the EISCAT radar.) One interpretation is as follows: Above a certain size such that the gravitational settling speed becomes more significant than the mean thermal speed, the aerosols would be height dependently ordered, with the larger ones sinking to the bottom. This would imply that the UHF PMSE are due to smaller aerosols, which would also likely mean less positive charge and greater number density. More extensive simultaneous measurements of PMSE with VHF and UHF radars need to be taken to investigate this possibility. A good altitude calibration is crucial in this regard. For example, turbulent scatter PMSE has not been completely ruled out for UHF radar; but if this is the dominant scatter mechanism, we would expect to see the echoes coming from the same altitude as the VHF PMSE, since the same aerosols would be responsible for enhancing the electron Schmidt number regardless of radar frequency. Height dependence of enhanced incoherent scatter spectral width could also, in principle, be used to obtain information on size versus height of charged aerosols. In this paper we presented calculations of spectral width versus aerosol radius which can be compared with such measurements.

On the theoretical side the greatest uncertainty regarding the validity of our ideas revolve around the importance of photoemission in the charging of aerosols. Unfortunately, without further experimental data on the material nature of these particles one cannot go much beyond speculation at this point.

Acknowledgments. We would like to thank Don Farley for making valuable comments on the initial draft of this paper and Dave Hysell for a turbulence modeling program. This work was funded by an NSF contract, ATM-8913153. Also, one of the authors (JYNC) was supported by a NASA Space Grant Fellowship.

\section{REFERENCES}

Auty, R. P., and R. H. Cole, Dielectric properties of ice and $\mathrm{D}_{2} \mathrm{O}$, J. Chem. Phys., 20, 1309-1314, 1952.

Balsley, B. B., and A. C. Riddle, Monthly mean values of the mesospheric wind field over Poker Flat, Alaska, J. Atmos. Sci., 41, 2368-2375, 1984.

Björn, L. G., E. Kopp, U. Herrmann, P. Eberhardt, P. H. G. Dickinson, D. J. Mackinnon, F. Arnold, G. Witt, A. Lundin, and D. B. Jenkins, Heavy ionospheric ions in the formation process of noctilucent clouds, J. Geophys. Res., 90, 7985-7998, 1985.

Böhnhardt, $H$., The charge of flufty dust grains of silicate and carbon near $\mathrm{P} / \mathrm{Halley}$ and $\mathrm{P} / \mathrm{Giacobini-Zinner,} \mathrm{Proc.} \mathrm{20th} E S$. LAB Symp. Halley's Comet, ESA SP-250, pp. 207-213, 1986.

Bronshtehn, V. A., and N. I. Grishin, Noctilucent Clouds, Keter, Jerusalem, Israel, 1976.

Chapman, S., and T. G. Cowling, The Mathematical Theory of Non-Uniform Gases, Cambridge University Press, New York, 1970.

Coy, L., D. C. Fritts, and J. Weinstock, The Stokes drift due to vertically propagating internal gravity waves in a compressible atmosphere, J. Atmos. Sci., 43, 2636-2643, 1986.
Czechowsky, P., I. M. Reid, R. Rüster, and G. Schmidt, VHF radar echoes observed in the summer and winter polar mesosphere over Andøya, Norway, J. Geophys. Res., 94, 5199-5217, 1989.

Dalgarno, A., M. R. C. McDowell, and A. Williams, The mobilities of ions in unlike gases, Philos. Trans. R. Soc., 250 , 413-425, 1958.

Dougherty, J. P., and D. T. Farley, A theory of incoherent scattering of radio waves by a plasma, 3, Scattering in a partly ionized gas, J. Geophys. Res., 68, 5473-5486, 1963.

Driscoll, R. J., and L. A. Kennedy, A model for the spectrum of passive scalars in an isotropic turbulence field, Phys. Fluids, $28,72-80,1985$.

Ecklund, W. L., and B. B. Balsley, Long-term observations of the Arctic mesosphere with the MST radar at Poker Flat, Alaska, J. Geophys. Res., 86, 7775-7780, 1981.

Farley, D. T., J. P. McClure, D. L. Sterling, and J. L. Green, Temperature and composition of the equatorial ionosphere, $J$. Geophys. Res., 72, 5837-5851, 1967.

Fukuyama, K., and W. Kofman, Incoherent scattering of an electromagnetic wave in the mesosphere: A theoretical consideration, J. Geomagn. Geoelectr., 32, 67-81, 1980.

Goertz, C. K., Dusty plasmas in the solar system, Rev. Geophys., $27,271,1989$.

Grams, G., and G. Fiocco, Equilibrium temperatures of spherical ice particles in the upper atmosphere and implications for noctilucent cloud formation, J. Geophys. Res., 82, 961-966, 1977.

Hagfors, T., Note on the scattering of electromagnetic waves from charged dust particles in a plasma, J. Atmos. Terr. Phys., in press, 1991.

Hall, C., Modification of the energy-wavenumber spectrum for heavy proton hydrates as tracers for isotropic turbulence at the summer mesopause, J. Geophys. Res., 95, 5549-5556, 1990.

Hall, C., and A. Brekke, High Schmidt numbers in the mesopause region from $224 \mathrm{MHz}$ incoherent backscatter, Geophys. Res. Lett., 15, 561-564, 1988.

Hall, T. M., J. Y. N. Cho, M. C. Kelley, and W. K. Hocking, A reevaluation of the Stokes drift in the polar summer mesosphere, J. Geophys. Res., this issue.

Havnes, O., U. de Angelis, R. Bingham, C. K. Goertz, G. E. Morfill, and V. Tsytovich, On the role of dust in the summer mesopause, J. Atmos. Terr. Phys., 52, 637-643, 1990.

Hill, R. J., Nonneutral and quasi-neutral diffusion of weakly ionized multiconstituent plasma, J. Geophys. Res., 83, 989-998, 1978.

Hill, R. J., and S. A. Bowhill, Small-scale fluctuations in D region ionization due to hydrodynamic turbulence, Aeron. Rep. 75, Aeron. Lab., Univ. of Ill., Urbana, 1976.

Inhester, B., J. C. Ulwick, J. Y. N. Cho, M. C. Kelley, and G. Schmidt, Consistency of rocket and radar electron density observations: Implication about the anisotropy of mesospheric turbulence, J. Atmos. Terr. Phys., 52, 855-873, 1990.

Kelley, M. C., The Earth's Ionosphere, Academic, San Diego, Calif., 1989.

Kelley, M. C., D. T. Farley, and J. Röttger, The effect of cluster ions on anomalous VHF backscatter from the summer polar mesosphere, Geophys. Res. Lett., 14, 1031-1034, 1987.

Kelley, M. C., J. C. Ulwick, J. Röttger, B. Inhester, T. Hall, and $T$. Blix, Intense turbulence in the polar mesosphere: Rocket and radar measurements, J. Atmos. Terr. Phys., 52, 875-891, 1990.

Michel, B., Ice Mechanics, University of Laval Press, Quebec, Canada, 1978.

Reid, G. C., Ice particles and electron "bite-outs" at the summer polar mesopause, J. Geophys. Res., 95, 13,891-13,896, 1990.

Röıtger, J., C. La Hoz, M. C. Kelley, U.-P. Hoppe, and C. Hall, The structure and dynamics of polar mesosphere surnmer echoes observed with the EISCAT 224-MHz radar, Geophys. Res. Lett., 15, 1353-1356, 1988.

Röttger, J., M. T. Rietveld, C. La Hoz, T. Hall, M. C. Kelley, and W. E. Swartz, Polar mesosphere summer echoes observed with the EISCAT 933-MHz radar and the CUPRI $46.9-\mathrm{MHz}$ radar, their similarity to-224 $\mathrm{MHz}$ radar echoes and their relation to turbulence and electron density profiles, Radio Sci., 25, 671687,1990 . 
Schunk, R. W., Transport equations for aeronomy, Planet. Space Sci., 23, 437-485, 1975.

Thomas, G. E., Solar Mesospheric Explorer measurements of polar mesospheric clouds (noctilucent clouds), J. Atmos. Terr. Phys., 46, 819-824, 1984.

Thomas, G. E., and J. J. Olivero, The heights of polar mesospheric clouds, Geophys. Res. Lett., 13, 1403-1406, 1986.

Tsytovich, V. N., U. de Angelis, and R. Bingham, Nonlinear transition scat tering of waves on charged dust particles in a plasma J. Plasma Phys., 42, 429-443, 1989.

Turco, R. P., O. B. Toon, R. C. Whitten, R. G. Keesee, and D. Hollenbach, Noctilucent clouds: Simulation studies of their genesis, properties and global influences, Planet. Space Sci., \$0, 1147-1181, 1982.
Ulwick, J. C., K. D. Baker, M. C. Kelley, B. B. Balsley, and W. L. Ecklund, Comparison of simultaneous MST radar and electron density probe measurements during STATE, J. Geophys. Res., 93, 6989-7000, 1988.

J. Y. N. Cho, T. M. Hall, and M. C. Kelley, School of Electrical Engineering, Engineering and Theory Center Bldg., Cornell University, Ithaca, NY 14853-3801.

(Received March 1, 1991; revised August 19, 1991; accepted October 15, 1991.) 